\title{
Synthesis of Coumarins Through Zinc Chloride-Catalyzed Hydroarylation of Acetylenic Esters by Phenols
}

\author{
Raquel A. C. Leão, Paula de F. de Moraes, Marcella C. B. C. Pedro and Paulo R. R. \\ Costa*
}

Laboratório de Química Bioorgânica (LQB), NPPN, CCS, Universidade Federal do Rio de Janeiro, Ilha da Cidade Universitária, 21941-590, Rio de Janeiro, RJ, Brazil. Fax: +552125626793

*prrcosta2011@gmail.com

Keywords: hydroarylation, zinc chloride, coumarins

\section{INTRODUCTION}

Coumarins comprise a class of natural products and many of them exhibit a broad range of biological activities. $^{1 a, b}$ Several routes have been described to

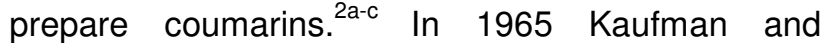
colworkers reported the hydroarylation of the ethyl propiolate (2a) by fluoroglucinol (1a) in the presence of stoichiometric amount of $\mathrm{ZnCl}_{2}$ leading to the coumarin $3 \mathbf{a}$ in good yield. ${ }^{2 a}$ The protocol reported by Kaufman was very few explored in the literature and no attempts to perform the hydroarylation in the presence of catalytic amounts of $\mathrm{ZnCl}_{2}$ was described. Herein we wish to report the zinc chloride-catalyzed hydroarylation of acetylenic esters 2 by phenols 1 leading to coumarins 3 .

\section{RESULTS AND DISCUSSION}

In order to know the scope of this reaction, phenols 1a-d were allowed to react with the acetylenic esters 2a-c in the presence of substoichiometric amount of $\mathrm{ZnCl}_{2}$ (Scheme 1).The hydroarylation of acetylenic ester $\mathbf{2 a}$ by the phenol $\mathbf{1 a}$ in the presence of $5 \mathrm{~mol}$ $\%$ of $\mathrm{ZnCl}_{2}$ at $100^{\circ} \mathrm{C}$ indeed led to the coumarin $3 a$ (Table 1, entry 1), as previously described in the literature, but this reaction is much more faster than reported by the authors, being complete in only five minutes. Under these conditions, propiolates $\mathbf{2} \mathbf{b}$ and 2c also reacted very fast with $\mathbf{1 a}$, leading to the coumarins $3 \mathbf{b}$ and $3 \mathbf{c}$ in excellent yields (entries 2 and 3). Coumarins $\mathbf{3 d - k}$, were also obtained in good yields (entries 4-11).

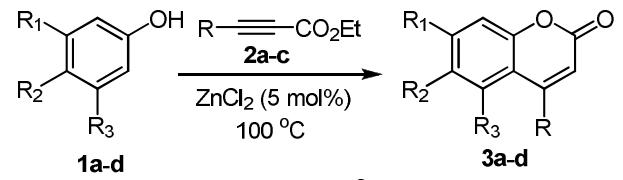

1a: $R_{1}=R_{3}=O H ; R_{2}=H \quad 2 a: R=H \quad$ 3a: $R_{1}=R_{3}=O H ; R_{2}=H ; R=H$ 1b: $R_{1}=R_{3}=O M e ; R_{2}=H \quad$ 2b: $R=M e ~ 3 b: R_{1}=R_{3}=O H ; R_{2}=H ; R=M e$ $\begin{array}{ll}\text { 1c: } R_{1}=O H ; R_{2}=R_{3}=H & \text { 2c: } R=P h \quad 3 c: R_{1}=R_{3}=O H ; R_{2}=H ; R=P h\end{array}$ 1d: $\mathrm{R}_{1}=\mathrm{R}_{2}=-\mathrm{OCH}_{2} \mathrm{O}-; \mathrm{R}_{3}=\mathrm{H} \quad$ 3d: $\mathrm{R}_{1}=\mathrm{R}_{3}=\mathrm{OMe} ; \mathrm{R}_{2}=\mathrm{H} ; \mathrm{R}=\mathrm{H}$ 3e: $R_{1}=R_{3}=O M e ; R_{2}=H: R=M e$ 3f: $R_{1}=R_{3}=O M e ; R_{2}=H ; R=P h$ 3g: $\mathrm{R}_{1}=\mathrm{OH} ; \mathrm{R}_{2}=\mathrm{R}_{3}=\mathrm{H} ; \mathrm{R}=\mathrm{H}$ 3h: $\mathrm{R}_{1}=\mathrm{OH} \cdot \mathrm{R}_{2}=\mathrm{R}_{3}=\mathrm{H} \cdot \mathrm{R}=\mathrm{M}$ 3i: $R_{1}=O H ; R_{2}=R_{3}=H ; R=M$ 3i: $\mathrm{R}_{1}=\mathrm{OH} ; \mathrm{R}_{2}=\mathrm{R}_{3}=\mathrm{H} ; \mathrm{R}=\mathrm{Ph}$ $\mathrm{R}_{3}=\mathrm{H} ; \mathrm{R}=\mathrm{H}$ $3 \mathbf{k}: \mathrm{R}_{1}=\mathrm{R}_{2}=-\mathrm{OCH}_{2} \mathrm{O}-; \mathrm{R}_{3}=\mathrm{H} ; \mathrm{R}=\mathrm{Me}$

Scheme 1. $\mathrm{ZnCl}_{2}$-promoted hydroarylation of acetylenic acids $2 \mathrm{a}-\mathbf{c}$ leading to the coumarins $3 \mathbf{a}-\mathbf{k}$.

$14^{\text {th }}$ Brazilian Meeting on Organic Synthesis $-14^{\text {th }}$ BMOS - September 01-05, 2011-Brasilia, Brazil
Table 1. $\mathrm{ZnCl}_{2}(5 \mathrm{~mol} \%)$ catalyzed synthesis of coumarins.

\begin{tabular}{|c|c|c|c|c|c|}
\hline Entry & $\mathbf{1}$ & $\mathbf{2}$ & $\mathbf{3}$ & Time & Yield (\%) \\
\hline 1 & $1 \mathrm{a}$ & $2 \mathrm{a}$ & $3 \mathrm{a}$ & $5 \mathrm{~min}$ & 88 \\
\hline 2 & $1 \mathrm{a}$ & $2 \mathrm{~b}$ & $3 \mathrm{~b}$ & $5 \mathrm{~min}$ & 84 \\
\hline 3 & $1 \mathrm{a}$ & $2 \mathrm{c}$ & $3 \mathrm{c}$ & $5 \mathrm{~min}$ & 95 \\
\hline 4 & $1 \mathrm{~b}$ & $2 \mathrm{a}$ & $3 \mathrm{~d}$ & $30 \mathrm{~min}$ & 73 \\
\hline 5 & $1 \mathrm{~b}$ & $2 \mathrm{~b}$ & $3 \mathrm{e}$ & $10 \mathrm{~min}$ & 77 \\
\hline 6 & $1 \mathrm{~b}$ & $2 \mathrm{c}$ & $3 \mathrm{f}$ & $12 \mathrm{~h}$ & 50 \\
\hline 7 & $1 \mathrm{c}$ & $2 \mathrm{a}$ & $3 \mathrm{~g}$ & $30 \mathrm{~min}$ & 85 \\
\hline 8 & $1 \mathrm{c}$ & $2 \mathrm{~b}$ & $3 \mathrm{~h}$ & $3 \mathrm{~h}$ & 88 \\
\hline 9 & $1 \mathrm{c}$ & $2 \mathrm{c}$ & $3 \mathrm{i}$ & $12 \mathrm{~h}$ & 54 \\
\hline 10 & $1 \mathrm{~d}$ & $2 \mathrm{a}$ & $3 \mathrm{j}$ & $1 \mathrm{~h}$ & 70 \\
\hline 11 & $1 \mathrm{~d}$ & $2 \mathrm{~b}$ & $3 \mathrm{k}$ & $10 \mathrm{~min}$ & 31 \\
\hline
\end{tabular}

\section{CONCLUSION}

In the presence of $5 \mathrm{~mol} \%$ of $\mathrm{ZnCl}_{2}$, activated phenols afforded coumarins in good yields. The method present herein lead to coumarins in better yields when compared to reported procedures based on Platina ${ }^{2 b}$ and Palladium ${ }^{2 \mathrm{c}}$ catalyzed hydroarylation of propiolates described recently and, in addition, is faster and less expensive.

\section{ACKNOWLEDGEMENTS}

We are indebted to FAPERJ, CNPq and CAPES

\section{REFERENCES}

a) Volz, N.; Toräng, J.; Hinz, S.; Bräseb, S.; Müllera, C. E. Bioorg. Med Chem. 2009, 17, 2842. b) Borges, F.; Roleira, F.; Milhazes, N.; Santana, L.; Uriarte, E. Curr. Med. Chem. 2005, 12, 887.

${ }^{2}$ a) Kaufman, K. D.; Kelly, R. C. Heterocycl. Chem. 1965, 2, 91. b) Oyamada, J.; Kitamura, T. Tetrahedron 2006, 62, 6918. c) Trost, B. M.; Toste, F. D.; Greenman, .K. J. Am. Chem. Soc. 2003, 125, 4518. 